\title{
What Is Being Trained? How Divergent Forms of Plasticity Compete To Shape Locomotor Recovery after Spinal Cord Injury
}

\author{
J. Russell Huie, ${ }^{1, \star}$ Kazuhito Morioka, ${ }^{1, *}$ Jenny Haefeli, ${ }^{1}$ and Adam R. Ferguson ${ }^{1,2}$
}

\begin{abstract}
Spinal cord injury (SCI) is a devastating syndrome that produces dysfunction in motor and sensory systems, manifesting as chronic paralysis, sensory changes, and pain disorders. The multi-faceted and heterogeneous nature of SCI has made effective rehabilitative strategies challenging. Work over the last 40 years has aimed to overcome these obstacles by harnessing the intrinsic plasticity of the spinal cord to improve functional locomotor recovery. Intensive training after SCI facilitates lower extremity function and has shown promise as a tool for retraining the spinal cord by engaging innate locomotor circuitry in the lumbar cord. As new training paradigms evolve, the importance of appropriate afferent input has emerged as a requirement for adaptive plasticity. The integration of kinematic, sensory, and loading force information must be closely monitored and carefully manipulated to optimize training outcomes. Inappropriate peripheral input may produce lasting maladaptive sensory and motor effects, such as central pain and spasticity. Thus, it is important to closely consider the type of afferent input the injured spinal cord receives. Here we review preclinical and clinical input parameters fostering adaptive plasticity, as well as those producing maladaptive plasticity that may undermine neurorehabilitative efforts. We differentiate between passive (hindlimb unloading [HU], limb immobilization) and active (peripheral nociception) forms of aberrant input. Furthermore, we discuss the timing of initiating exposure to afferent input after SCI for promoting functional locomotor recovery. We conclude by presenting a candidate rapid synaptic mechanism for maladaptive plasticity after SCI, offering a pharmacological target for restoring the capacity for adaptive spinal plasticity in real time.
\end{abstract}

Keywords: neuroplasticity; recovery; rehabilitation; spinal cord injury

\section{Introduction}

Q PINAl CORD INJURY (SCI) is a devastating, multi-faceted disorder that affects 250,000 people in the United States each year. ${ }^{1}$ SCI produces a number of behavioral and physiological consequences, notably limb paralysis below the injury and dysfunction of sensory and autonomic systems. Recent exciting findings in both preclinical and clinical SCI have challenged the historical perspective that little functional recovery could occur after SCI. ${ }^{2-4}$ It is now clear that the injured spinal cord has the capacity for numerous forms of plasticity including formal learning and memory.

Preclinical and clinical work over the past 40 years has shown that the spinal cord is highly sensitive to training, and even exhibits simple forms of classically defined learning. ${ }^{4-10}$ The promise of functional locomotor recovery has led to an ever-growing list of preclinical injury models, training paradigms, and increasingly sophisticated outcome metrics that take into account the multi- dimensional nature of SCI recovery. ${ }^{11-13}$ Preclinical experiments detailing the benefits of intensive locomotor training to facilitate lower extremity function after SCI have influenced clinical neurorehabilitation methods with promising results., ${ }^{4,10,14}$

This work has largely focused on promoting appropriate afferent input for effective neurorehabilitation, but there has been relatively little focus on the alternative potential that aberrant or inappropriate afferent input may induce maladaptive plasticity. Here we review the evolving optimization parameters for effective adaptive training, as well as recent work detailing the untoward neurobiological consequences of inappropriate afferent input on spinal motor plasticity after SCI.

\section{Adaptive Locomotor Neurorehabilitation}

A growing body of work from both animal models and human SCI has shown that effective neurorehabilitation requires a precisely tuned interaction between peripheral input and spinal motor

\footnotetext{
${ }^{1}$ Department of Neurological Surgery, Brain and Spinal Injury Center, University of California, San Francisco, California.

${ }^{2}$ San Francisco Veterans Affairs Medical Center, San Francisco, California.

*The first two authors contributed equally.
} 
circuits. ${ }^{5,8,10,15}$ Adaptive spinal training is optimized when there is adequate and appropriate afferent input modulating spinal circuitry, such as the central pattern generator (CPG), that can generate rhythmic locomotor activities with proprioceptive and sensory feedback.

\section{Adaptive neurorehabilitation in SCl animal models}

Work on the neural control of locomotion began over 100 years ago, with Thomas Graham Brown and Charles Sherrington demonstrating, in decerebrate cats, that stepping behavior could be elicited independent of supraspinal control. ${ }^{16-18}$ Although early experiments to engage spinal locomotor circuitry were successful in producing rhythmic stepping behavior without afferent input in spinalized cats, they fell short of improving functional overground locomotor recovery. ${ }^{16-22}$ Ambulatory training studies of functional CPG mechanisms have demonstrated the importance of afferent inputs during locomotion for improving functional locomotor recovery in spinalized cats. ${ }^{23-26}$ Partial body-weight bearing/loading/unloading conditions during ambulatory training are required to improve impaired stepping movements due to the muscle weakness in hindlimbs after SCI. ${ }^{8}$ Consequently, the introduction of partial body-weight support and tailoring the amount of support to the individual subject's recovery, has led to lasting alterations in locomotor output, and gradual recovery of weight-supported stepping. ${ }^{6,23,27-29}$ Human and animal studies have shown a direct increase in the magnitude of limb muscle activity, weight-bearing load, and electrophysiological neuromotor engagement with repeated training, suggesting that adaptive spinal plasticity relies on loading-related afferent input during locomotion. ${ }^{30-32}$ Locomotor training has been shown in a number of models to be quite task-specific, with the form of functional recovery occurring as an activity-dependent process. ${ }^{33-35}$ For example, early work in spinalized cats showed that although step training could produce improvements in locomotion, it did not improve standing. Conversely, those cats given a specific stand-training regimen showed improved standing but little increase in locomotor ability compared with the step-trained cats. ${ }^{5}$ This specificity suggests that simply reintroducing loading-related afferent input is not sufficient to produce broad adaptive locomotor recovery; the source of the afferent input, proprioceptive feedback, and multi-level central nervous system (CNS) synaptic integration within the spinal cord are crucial in determining the quality of the training effect. The spinal cord integrates afferent input, including cutaneous sensory input, proprioceptive signals, and kinematic information in a highly dynamic fashion. Forssberg and colleagues observed that stimulation of the dorsal surface of the spinalized cat hindpaw could elicit either flexion or extension response depending on the step phase ("reflex reversal"). ${ }^{36,37}$ This type of state-dependent motor output reinforces the notion that the injured spinal cord has a great capacity for adaptive training, given the appropriate afferent input. Importantly, Bouyer and Rossignol demonstrated the necessity for afferent input in treadmill training, showing that at least one cutaneous afferent nerve must remain intact to produce appropriate plantar foot placement and weight-bearing in spinalized cats. ${ }^{38}$ The capacity for dynamic adaptive traning was elegantly illustrated by Timoszyk and colleagues in a set of experiments aimed at optimizing locomotor training. Using a robotic system to fully control all training parameters, they found that by applying slight downward force on one hindlimb during the stance phase of locomotion on a treadmill produced not only an adaptation of the stance duration in the affected leg, but also a modulation in the swing time of the contralateral hindlimb to preserve stepping symmetry. ${ }^{39}$ Such a finding highlights both the importance for somatosensory feedback as well as the central organization of adaptive spinal plasticity.
Others have recently developed rodent locomotor training and assessment models that are specifically designed with the importance of somatosensory input in mind. These models allow for quadrupedal stepping, as opposed to the bipedal training that is standard for most body-weight supported treadmill training strategies. Shah and colleagues found that quadrupedal locomotor training was more effective in improving hindlimb function than bipedal training. ${ }^{40}$ These findings support previous work showing that sensory and proprioceptive input from forelimbs are significant contributors to the generation of hindlimb stepping in at least some quadrupeds. ${ }^{41}$ Ward and colleagues recently demonstrated that treadmill training of the forelimbs alone does not improve locomotor recovery, but quadrapedal training does. ${ }^{42}$ Other researchers have recently foregone the treadmill altogether, in favor of overground stepping, with the goal of providing a more natural locomotor environment that would presumably provide a more comprehensive set of sensory, proprioceptive, and kinematic inputs that would take full advantage of the integrative properties of the spinal cord to better induce adaptive plasticity through both afferent and efferent synapses on the spinal cord. ${ }^{43,44}$

\section{Adaptive neurorehabilitation in humans with $\mathrm{SCl}$}

The importance of appropriate afferent input and adequate proprioceptive feedback extends from the bench to bedside. Dietz and colleagues observed that locomotor training in which individuals with clinically complete SCI were fully unloaded was not sufficient to induce significant leg muscle activation; however coordinated stepping movements and muscle activity can be induced by bodyweight support and standing on a treadmill. ${ }^{8,45}$ Extensive training with partial body-weight support allows for a gradual increase in loading that correlated with increased electromyogram (EMG) magnitude, similar to those seen in preclinical models. ${ }^{30,31}$ Gradual reloading is just one component of successful adaptive locomotor training. As seen in animal models, the integration of multi-modal sensory input appears to be necessary for effective neurorehabilitation. ${ }^{46}$ Clinical evidence suggests that loading, treadmill speed, and kinematic input are key parameters that must be tailored for each individual patient to provide appropriate proprioceptive feedback and optimize the effects of locomotor training. ${ }^{14}$

Recently, some groups have tested robot-assisted treadmill training to provide appropriate kinematic input. ${ }^{47-49}$ Such innovations aim to cut the manual labor and cost related to neurorehabilitation, and increase the amount of quantitative information that can be used to provide more precise and accurate training parameters. Robotassisted treadmill training has been shown to produce some beneficial effects, especially in patients with severe SCI-induced deficits. ${ }^{50}$ Yet, evidence from randomized controlled studies comparing robotic and manual treadmill assistance demonstrate that manual treadmill training outperforms robotic-assisted training. ${ }^{51,52}$ This implies that active participation is a crucial element for adaptive neurorehabilitation, and robotic-assisted treadmill training alone may be too passive to induce lasting training effects on adaptive spinal cord plasticity and locomotor recovery.

The last decade has also seen the rise and growth of overground robotic exoskeleton orthosis systems. These systems have shown great promise as a tool to restore patient independence, allowing patients with a SCI to stand and ambulate using varying degrees of automaticity. Although these benefits are clear, it is unknown how these systems are engaging or affecting spinal cord plasticity. Two types of powered exoskeleton orthoses that are U.S. Food and Drug Administration (FDA)-approved (Re-Walk ${ }^{\mathrm{TM}}$ and Indego ${ }^{\circledR}$ ) require no active leg movement from the user and thus may not be 
suitable as rehabilitative treatments. In contrast, the $\mathrm{EKSO}^{\circledR}$ system has been approved for rehabilitation treatment in which gradations of active limb movement can occur. ${ }^{53}$ The treadmill findings for both preclinical studies and clinical rehabilitation trials would suggest that the inclusion of an active user component may be critical for lasting adaptive neuroplasticity to occur. Future work will be necessary to determine the extent and quality of neuroplasticity that might be elicited by these promising robotic overground systems.

\section{Neuromodulatory drugs and stimulation technologies to boost adaptive spinal cord plasticity}

Apart from locomotor training, researchers have also sought to induce and/or potentiate locomotion by directly modulating the excitability of spinal neurons. One method researchers have used to increase excitability in the injured spinal cord is through pharmacological interventions to alter locomotor function. Early work from Jankwoska and others identified the noradrenergic system as having a neuromodulatory effect on locomotor circuitry, showing that dopamine precursor L-DOPA induced excitation in spinal motor neurons and initiated rhythmic hindlimb movement in the spinalized cat. ${ }^{27,54}$ The alpha2 adrenergic agonist clonidine was also shown to effectively potentiate stepping for spinalized cats on a treadmill, with a single injection improving stepping for up to $6 \mathrm{~h} .{ }^{55}$ Interestingly, when daily clonidine administration was followed by intensive treadmill training, the locomotor training effects persisted for days after the drug administration was stopped. ${ }^{55}$ Similarly, serotonergic agonists such as quipazine have been used to boost spinal excitability in rat models of complete SCI, resulting in improved alternating stepping patterns, ${ }^{56}$ as well as pronounced improvements in the swing, and extension phases of stepping, and plantar paw placement. ${ }^{57}$ Importantly, in contrast with noradrenergic interventions, quipazine has not been shown to induce air stepping in other animal models, indicating that this system may potentiate existing locomotor patterns rather than initiate pattern generation. 57,58

Recent promising therapeutic strategies have aimed to take advantage of the integrative characteristics of spinal motor circuitry, by taking a multi-system approach to facilitating locomotion. Interventions that combine loading-related afferent input from locomotor training with a boost in spinal excitability from both tonic epidural electrical stimulation and quipazine administration have produced remarkable locomotor recovery. ${ }^{59}$ Similarly, Harkema and colleagues have combined body-weight supported locomotor training with epidural stimulation in a small cohort of human patients with clinically complete SCI. ${ }^{4,10}$ They found that following intensive stand training, the introduction of tonic epidural stimulation was sufficient to enable voluntary lower limb movement. These findings suggest that even with extremely limited sparing, supraspinal control can be accessed given the appropriate modulation of the state of excitability in spinal motor systems. Although these interventions show great promise, it will be important in the future to carefully tune the input parameters to promote adaptive plasticity. These recent findings also highlight how sensitive the injured spinal circuitry is to changes in excitability. As we discuss in the following section, this potential for plasticity also leaves the spinal cord vulnerable to maladaptive changes.

\section{Maladaptive Plasticity}

The question of the timing and specific afferent input required for precision neurorehabilitation after SCI remains open. Despite many examples of successful neurorehabilitative efforts to reengage locomotor activity, evidence suggests that neurorehabilitation in current settings has limited effectiveness. ${ }^{60}$ Maladaptive forms of plasticity such as hyper-reflexia/spasticity and chronic central pain can emerge spontaneously after SCI, ${ }^{61-64}$ and once established, maladaptive spinal cord plasticity may undermine the effectiveness of common forms of adaptive rehabilitation including stand training and swimming therapy. ${ }^{65,66}$ Accumulating evidence suggests that afferent input in the acute phase of injury can also contribute to maladaptive forms of plasticity, thus making attempts at later adaptive training more difficult. ${ }^{66-71}$ Although systematic review suggests that partial weight-bearing training initiated from 1 to 2 weeks of an 8-week recovery period after SCI results in positive outcomes, both extreme overuse and complete disuse of the impaired forelimb early after CNS injury can reduce functional locomotor recovery. ${ }^{72,73}$ Thus, the benefit of early onset training remains controversial. Establishing precision neurorehabilitation requires identifying the limited time window of fruitful adaptive plasticity and the difference between appropriate and inappropriate afferent input for promoting locomotor recovery ${ }^{74,75}$ In the following section we will focus on the contributing role afferent input may play in use-dependent maladaptive plasticity after SCI.

\section{Limb immobilization and hindlimb unloading}

As paralysis is a primary consequence of SCI, the muscle atrophy and weakness in hind-/lower-limb resulting from severe SCI or long-term immobilization reduces muscle afferent input, and may likely lead to locomotor dysfunction due to disuse. Although musculoskeletal plasticity can occur in mild-to-moderate SCI and can be facilitated by ambulatory training, the long-term limb immobilization just after severe SCI prevents plasticity from contributing to recovery of muscles. ${ }^{76-80}$ Whereas much attention has been given to the effects of SCI-induced limb immobilization on musculoskeletal plasticity, ${ }^{81}$ a number of preclinical studies have shown that maladaptive spinal plasticity produced by inappropriate afferent input can play a pivotal role in undermining future locomotor recovery, developing hyper-reflexia, spasticity, and intractable pain. ${ }^{82,83}$ Preclinical work by Caudle and colleagues has shown that in a wheelchair model of acute limb immobilization after SCI, immobilized rats show significantly less locomotor recovery if compared with non-immobilized rats as control. ${ }^{84}$ In healthy human subjects immobilization can induce substantial maladaptive spinal plasticity contributing to transient neurological dysfunction.

Another model that has been used to investigate the mechanisms of altered afferent input is the hindlimb unloading (HU)/hindlimb suspension (HLS, HS) model developed by Morey-Holton and Globus. ${ }^{85,86} \mathrm{HU}$ affects sensory afferent signals and induces an increase in neuronal excitation of the spinal cord. Although this model has been most commonly used in intact rats as a means to study the effects of microgravity during space flight, the results from these studies may be relevant for SCI research. ${ }^{87}$ HU greatly reduces muscle spindle afferent input, and this perturbation in afferent input has a robust effect on lumbar spinal circuitry. ${ }^{88,89}$ Given that muscle spindle feedback has been shown to drive adaptive plasticity of propriospinal relay circuits and recovery after incomplete SCI, it could be hypothesized that HU would have a deleterious impact on recovery after SCI. ${ }^{90}$ The Hoffmann reflex (H-reflex) test of motorneuron pool excitability is a useful tool for assessing the modification of spinal plasticity, and the anti-gravity slow-twitch soleus muscle is well-recognized as an appropriate 
muscle for recording the modification of the H-reflex at the L5 ventral root level after HU. ${ }^{91-93}$ It has been shown that the H-reflex has lowered thresholds after 3 weeks of $\mathrm{HU}$ and a similar observation correlates with reduced soma size in lumbar alphamotor neurons after 2 weeks of HU, suggesting changes in motorneuron excitability. ${ }^{91,92}$ Treadmill training of intact rats that had previously undergone 2 weeks of $\mathrm{HU}$ showed robust alterations in neuromuscular pattern during locomotion and a broad disorganization of locomotor performance, including increased cycle duration, frequent hyperextensions of the ankle, and lateral instability. ${ }^{94}$ These rats also exhibited locomotor deficits similar to those seen previously by Lovely and colleagues in spinalized cats, ${ }^{5}$ that is, paw drag at the start of swing phase and lack of hindlimb equilibrium, suggesting that $\mathrm{HU}$, even in the intact animal, induced spinal cord changes that were influenced by both the lack of afferent information and altered supraspinal input. Clinical studies have shown that both long-term prolonged bed rest without traumatic CNS injury and microgravity in spaceflight may induce slower axonal conduction velocity in the branching axon terminals. ${ }^{95}$ Furthermore, 4-week lower extremity unloading without traumatic CNS injury induces substantial maladaptive spinal plasticity contributing transient neurological dysfunction, suggesting that the muscle deconditioning due to disuse decreases afferent input and proprioceptive feedback in consequence of adaptation to unloading condition. ${ }^{96}$ While these findings suggest that hindlimb unloading may induce locomotor impairments, little is known about the direct effect of HU on spinal plasticity after SCI. Recent preclinical studies suggest that early HU after mild-to-moderate SCI impairs long-term locomotor recovery, an effect that may be mediated by maladaptive synaptic plasticity. ${ }^{97}$ Future experiments will be crucial to determine the neurobiological mechanisms by which $\mathrm{HU}$ may alter motor excitability and spinal plasticity under the pathological conditions of SCI.

\section{Nociceptive/noxious afferent input}

Although it is well-established that SCI itself is sufficient to produce sensory dysfunction such as neuropathic pain, the role of peripheral nociceptive input in shaping maladaptive spinal cord plasticity is less well-known. Epidemiological studies reveal a high incidence of concomitant peripheral injury in the clinical SCI population, ranging from fractured limbs, lacerations, and abrasions, to peripheral nerve injuries and nociceptor sensitization. ${ }^{98-101}$ It will be of great clinical importance to understand the potential for peripheral nociceptive input to modulate sensory and motor plasticity in the injured spinal cord. A growing body of literature from the pain research field has indicated that unmitigated nociceptive input can produce robust alterations in sensory function in the intact spinal cord. The mechanisms of synaptic plasticity underlying these changes have been shown to be akin to those seen in hippocampal learning and memory. ${ }^{102-104}$ As such, spinal cord changes produced by tetanic nociceptive stimulation have been referred to as "pain memory traces," and have been suggested to be the source of sensory dysfunction including hyper-reflexia, hyperalgesia, and neuropathic pain. ${ }^{105-108}$ In the context of SCI, intense peripheral nociceptive input has been shown to affect not only sensory systems, but motor plasticity as well. ${ }^{82,109-113}$

To test the effect of nociceptive input on sensory and motor recovery, Grau and colleagues produced a thoracic spinal contusion injury in rats, followed by administration of an experimentally controlled uncontrollable nociceptive stimulus (electrical shock to the tail sufficient to drive $\mathrm{C}$-fiber activation). They found that as little as 6 min of C-fiber-strength intermittent nociceptive stimulation could induce sensory dysfunction (hyperalgesia and mechanical allodynia), produce acute learning deficits on an adaptive motor training task, and undermine locomotor recovery for at least 6 weeks. ${ }^{82}$ A recent clinical case study has similarly shown that healthy human subjects given brief peripheral nociceptive input are unable to retain a locomotor learning task. ${ }^{14}$ Together these observations suggest that peripheral nociceptive stimulation early after SCI produces lasting changes within the spinal cord circuitry that undermine future neurorehabilitation efficacy. Targeting the synaptic mechanisms of these intraspinal changes has the potential to reverse maladaptive plasticity for improved locomotor recovery.

\section{A synaptic mechanism of rapid-induction maladaptive plasticity in $\mathrm{SCl}$}

Little published literature has explicitly tested the impact of nociceptive stimulation on rapid synaptic plasticity in spinal cord motor circuits after SCI. In an attempt to uncover a pharmacological target for stimulus-dependent maladaptive plasticity in SCI, our team recently tested the impact of below-level nociceptive stimulation on ventral spinal motor neurons after SCI, using the same uncontrollable C-fiber-strength electronociceptive stimulation paradigm extensively described by Grau and colleagues. ${ }^{82,115-118}$ Our hypothesis was that aberrant stimulation would generate intrinsic ventral horn motorneuron changes after SCI similar to those known to be involved in maladaptive sensory plasticity in the dorsal horn in pain syndromes. Pain plasticity has been shown to be mediated by rapid alterations in synaptic AMPA receptor (AMPAR) levels and phosphorylation similar to those observed in brain-dependent learning and memory. ${ }^{119,120}$ Motor neurons are highly sensitive to glutamatergic overdrive following SCI due to rapid trafficking of AMPARs from intracellular stores to the motorneuron membrane demonstrating that a mechanism of synaptic strengthening actually contributes to SCI pathology. ${ }^{121}$ In addition, this effect was specifically associated with an abnormal increase in GluA2-lacking, calcium-permeable AMPARs (CP-AMPARs). We reasoned that maladaptive spinal motor plasticity induced by peripheral nociceptive input might also reflect an overdrive of CPAMPARs on motorneuron dendrites and somata after SCI, reflecting a global hyperexcitability throughout the injured spinal cord. Further, because calcium is a positive regulator of rapid AMPAR trafficking to synapses, this calcium permeability could set up a positive feedback loop of ever-increasing synaptic excitability. Supporting this, nociceptive input after complete SCI increased the ratio of synaptoneurosomal GluA1 to GluA2 AMPAR subunits in the ventral lumbar spinal cord within 20 min of nociceptive stimulation (the earliest time-point tested), indicating an acute shift toward increased CP-AMPAR expression. High-resolution three dimensional (3D) confocal imaging revealed time-dependent increases in GluA1 and decreased GluA2 AMPA receptor subunit expression on large spinal motorneurons after nociceptive input (Fig. 1), with GluA2 removal occurring first at extrasynaptic sites followed by synaptic removal $2 \mathrm{~h}$ later. ${ }^{118}$

Prior work has linked rapid trafficking of CP-AMPARs to plasma membrane to early excitotoxic cell death in the models of stroke, traumatic brain injury, and SCI. ${ }^{121-123}$ Does nociceptive stimulation produce dysfunction simply by enhancing this AMPAR-dependent excitotoxic cell death below SCI? Quantitative biochemistry of cell death markers c-jun, caspase 3, and calpain I suggest that the answer is no-nociception does not increase cell death below SCI. ${ }^{118}$ The implication of this finding is that nociception-induced dysfunction 
A

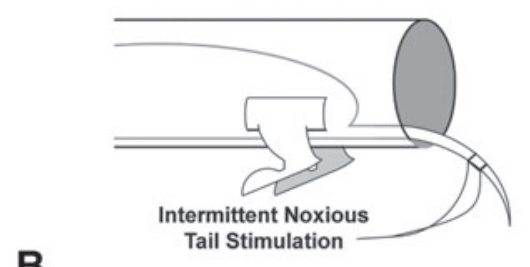

B

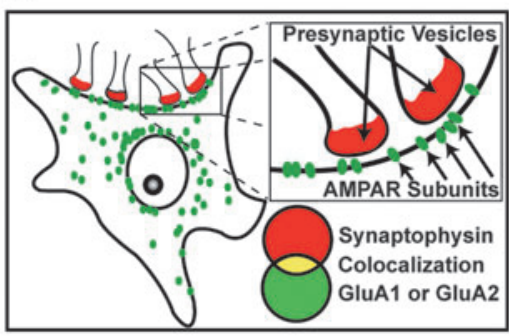

C
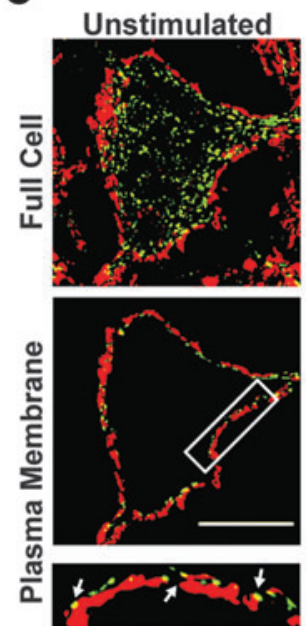

GLUA1
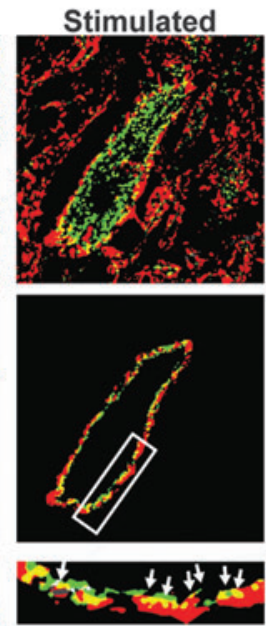

D

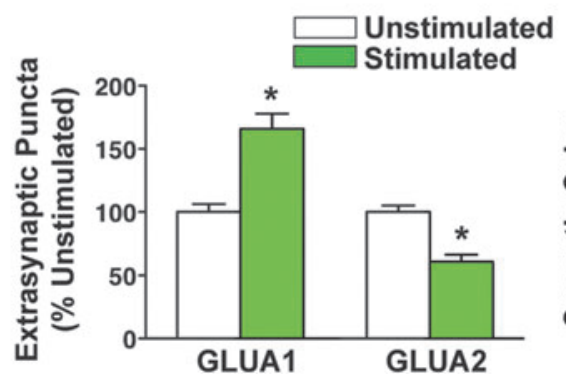

GLUA2
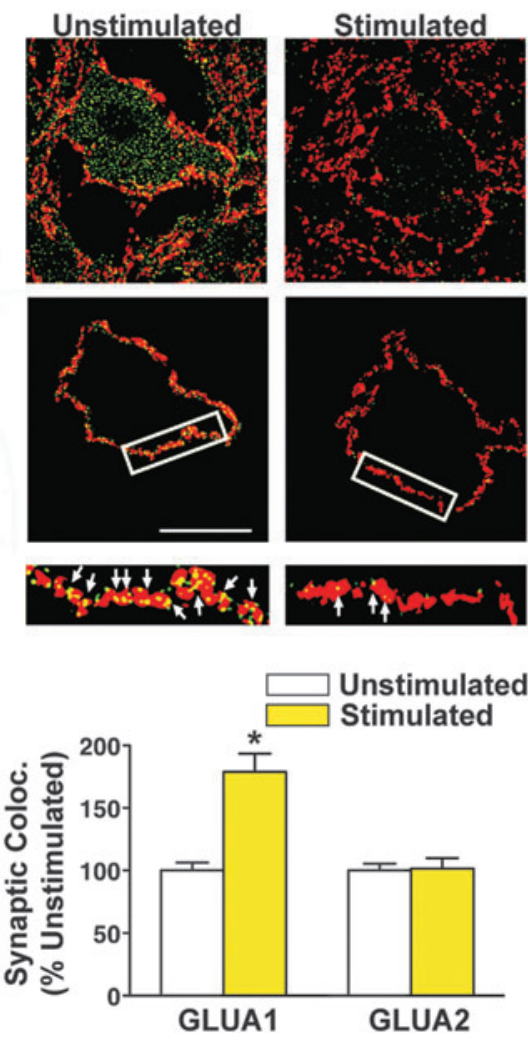

FIG. 1. Peripheral nociceptive input alters AMPA receptor plasticity on motor neurons after complete SCI. (A) Nociceptive input was delivered by electrical stimulation to the tail of male rats that had received a complete spinal transection at the second thoracic vertebrae. Stimulation delivery was computer-controlled, and administered intermittently over the course of 6 min. (B) Optical detection of synaptic AMPA receptor subunits. Immunofluorescent expression of presynaptic marker (red, synaptophysin) and AMPA receptor subunit (green, GluA1 or GluA2) was assessed, as well as the colocalization of red and green puncta (yellow, colocalization), indicating synaptic AMPA receptor subunit expression. (C) Large L4-5 ventral horn neurons were assessed for colocalization of GluA1/2 and synaptophysin-positive synapses after nociceptive stimulation. Upper images show full neuropil area for a single confocal plane ("Full Cell"); lower images show a digitally isolated 2- $\mu$ m wide cutout of the plasma membrane area in the single confocal plane ("Plasma Membrane"). (D) Extrasynaptic GluA1 was significantly increased 20 min after stimulation, whereas extrasynaptic GluA2 is significantly decreased $(* p<0.05)$. Synaptic colocalization of GluA1 and synaptophysin was also significantly increased $(* p<0.05)$, whereas synaptic GluA2/synaptophysin colocalization is unaltered by stimulation. These findings suggest a rapid increase in synaptic GluA2lacking AMPA receptors in response to nociceptive input. Modified from Huie et al. ${ }^{118}$ SCI, spinal cord injury.

may be reversible. Supporting this, delayed intrathecal treatment with a specific antagonist to CP-AMPARs therapeutically reverses nociception-induced maladaptive plasticity, rescuing the capacity for adaptive motor learning. ${ }^{118}$ These findings identify a specific synaptic mechanism for maladaptive spinal cord plasticity induced by peripheral input, and suggest a unique therapeutic target that may help to restore the capacity for future adaptive modifications. This proof-of-concept study suggests that selective CP-AMPAR antagonists early after injury could boost adaptive neurorehabilitation by blocking maladaptive plasticity.

Future work will be necessary to determine if regulating AMPAR activity is an effective method for restoring the capacity for adaptive plasticity in chronic SCI. Moderate AMPAR-mediated calcium influx can be beneficial and necessary for adaptive plasticity (as in the stabilization of long-term potentiation in the brain), but as CPAMPAR activity increases in response to insult or nociceptive input, an excitatory saturation level is reached, at which point spinal neurons may lose the capacity to encode more subtle task-specific simulation patterns, as in the adaptive spinal training task (Fig. 2).

Figure 2 introduces the idea that SCI places the spinal cord into a new state of metaplasticity where the threshold for inducing plas- ticity in local segmental circuitry is altered after loss of descending control from the brain and can move into either an adaptive or maladaptive direction. This idea of CNS metaplasticity was first advanced in the hippocampal and cortical plasticity literature and has been shown to involve modulation of AMPAR by upstream signaling pathways including metabotropic glutamate receptor activation of calcium-dependent intracellular pathways, brainderived neurotrophic factor, and other mechanisms. ${ }^{124-127}$ Several groups have subsequently demonstrated the existence of metaplasticity in spinal cord locomotor circuitry below SCI. ${ }^{128-132}$ Understanding spinal cord (re)-training potential as a form of metaplasticity provides a fertile literature to draw from for pharmacological targets to carefully tune and improve spinal cord training after injury.

\section{Rapid-induction maladaptive plasticity: a potential therapeutic target}

Therapeutic interventions to regulate glutamatergic overdrive following injury or insult will likely be key in promoting adaptive plasticity. AMPAR antagonists have shown efficacy in restoring 


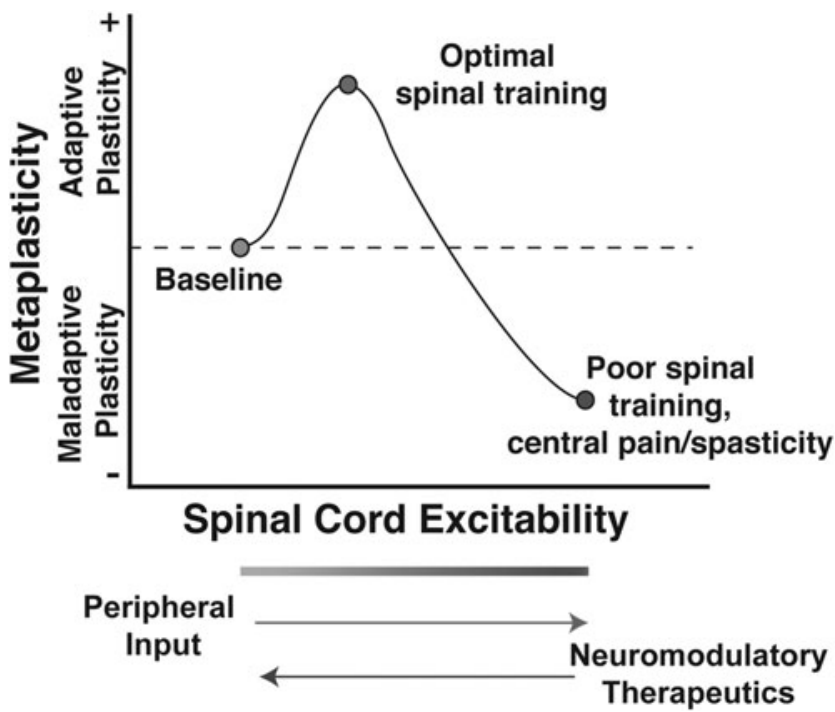

FIG. 2. Theoretical model of the effect of peripheral input on the metaplasticity of spinal function after SCI. Appropriate peripheral sensory and proprioceptive input after SCI can strengthen excitatory tone to improve the capacity for adaptive spinal training. However, unmitigated peripheral input may overdrive excitability, resulting in synaptic saturation that induces maladaptive locomotor and sensory plasticity (e.g., impaired stepping, spasticity, chronic neuropathic pain). SCI places spinal cord circuitry into an unstable metaplastic state where spinal cord plasticity can take on either adaptive or maladaptive forms. From this perspective, therapeutic interventions aimed at re-tuning synaptic strength toward optimal adaptive plasticity, while limiting maladaptive plasticity, will be essential for improving functional recovery. Modified from Huie et al. ${ }^{118}$ SCI, spinal cord injury.

adaptive neural function in a number of neuropathological models, including epilepsy, ischemia, neuropathic pain, opioid-induced hypersensitivity, and motor neuron diseases. ${ }^{122,133-140}$ However, the SCI field has been less focused on balancing homeostatic glutamatergic plasticity, and most neuromodulatory approaches have instead focused on driving locomotor activity through pharmacological and neurostimulator technologies (see section on neuromodulation, above). It is important to note that although locomotor training models that use neurostimulation (e.g., tail stimulation in spinalized rats) to facilitate stepping have yielded promising results, the potential for robust afferent input to detrimentally alter sensory and motor function must be considered. As these approaches to increase spinal synaptic connectivity continue to yield gains in function, the optimal tuning of homeostatic plasticity is likely to become a relevant target for precision neurorehabilitation. ${ }^{141}$

\section{Conclusions and Future Considerations}

Effective adaptive training to promote functional locomotor recovery requires highly tuned, task-specific models. Conversely, aberrant peripheral input, whether in passive forms such as limb immobilization or unloading, or active forms such as peripheral nociceptive input, appear to broadly affect both sensory and motor systems that can induce lasting maladaptive plasticity that may reflect glutamatergic dysregulation in the injured spinal cord. A number of critical questions need to be answered:

1. How might early inappropriate afferent input affect longterm synaptic plasticity? Could these early mechanisms persist, becoming forces we must overcome in chronic SCI? Given the high incidence of neuropathic pain in the SCI population, it may be possible that early input (due to concomitant peripheral injury or abnormal spindle feedback) could be engaging maladaptive sensory plasticity that manifests as neuropathic sensory dysfunction in the chronic stage. Thus early interventions to alleviate or block the development of maladaptive plasticity will be crucial.

2. Are passive, but abnormal forms of afferent input such as HU or limb immobilization in the acute phase of SCI sufficient to induce maladaptive alterations in spinal circuitry that persist into the chronic phase? Recent studies have established a synaptic mechanism for maladaptive plasticity in lumbar motor neurons following nociceptive input in a complete SCI model, but it remains an open question whether similar changes occur in incomplete contusion models, as well as in response to a more subtle form of inappropriate afferent input, such as HU. Likewise, given the limited efficacy of some robotic-assistance models, might the lack of active participation in such systems be producing passive abnormal afferent input that could induce maladaptive plasticity and hinder locomotor recovery? The answers to these questions could have major implications for the way we approach neurorehabilitation in general, and sensitivity to inappropriate afferent input in particular.

The work reviewed here highlights the challenges we face in developing effective neurorehabilitation strategies. In our efforts to induce adaptive plasticity, we must be aware of maladaptive alterations that must be overcome. The spinal cord is very plastic. This is a promising fact, but must be met with caution: there are divergent forms of plasticity, and it is clear the injured spinal cord is highly susceptible to both. Our goal must be to shape spinal plasticity toward an adaptive form while minimizing maladaptive plasticity.

\section{Acknowledgments}

This work was supported by National Institutes of Health (NIH) Grants NS069537, NS088475, NS067092 (Adam R. Ferguson); Wings for Life Spinal Cord Research Foundation Grants WFLUS008/12, WFLUS006/14 (Adam R. Ferguson) and WFLUS013/13 (Kazuhito Morioka); Craig H. Neilsen Foundation 224308 (Adam R. Ferguson) and 313739 (Jenny Haefeli); Japan Society for the Promotion of Science (JSPS) KAKENHI Grants 21800092 and 23700659 (Kazuhito Morioka); and Mitsui Sumitomo Insurance Welfare Foundation Research Grant (Kazuhito Morioka).

\section{Author Disclosure Statement}

No competing financial interests exist.

\section{References}

1. Dobkin, B.H. (2003). The Clinical Science of Neurologic Rehabilitation. Oxford University Press, $1 \mathrm{p}$.

2. van den Brand, R., Mignardot, J.-B., von Zitzewitz, J., Le Goff, C., Fumeaux, N., Wagner, F., Capogrosso, M., Martin Moraud, E., Micera, S., Schurch, B., Curt, A., Carda, S., Bloch, J., and Courtine, G. (2015). Neuroprosthetic technologies to augment the impact of neurorehabilitation after spinal cord injury. Ann. Phys. Rehabil. Med. 58, 232-237.

3. Lu, P., Wang, Y., Graham, L., McHale, K., Gao, M., Wu, D., Brock, J., Blesch, A., Rosenzweig, E.S., Havton, L.A., Zheng, B., Conner, J.M., Marsala, M., and Tuszynski, M.H. (2012). Long-distance 
growth and connectivity of neural stem cells after severe spinal cord injury. Cell 150, 1264-1273.

4. Angeli, C.A., Edgerton, V.R., Gerasimenko, Y.P., and Harkema, S.J. (2014). Altering spinal cord excitability enables voluntary movements after chronic complete paralysis in humans. Brain 137, 13941409.

5. Lovely, R.G., Gregor, R.J., Roy, R.R., and Edgerton, V.R. (1986). Effects of training on the recovery of full-weight-bearing stepping in the adult spinal cat. Exp. Neurol. 92, 421-435.

6. Wernig, A., and Müller, S. (1992). Laufband locomotion with body weight support improved walking in persons with severe spinal cord injuries. Paraplegia 30, 229-238.

7. Raineteau, O., and Schwab, M.E. (2001). Plasticity of motor systems after incomplete spinal cord injury. Nat. Rev. Neurosci. 2, 263-273.

8. Dietz, V., Müller, R., and Colombo, G. (2002). Locomotor activity in spinal man: significance of afferent input from joint and load receptors. Brain 125, 2626-2634.

9. Grau, J.W., Crown, E.D., Ferguson, A.R., Washburn, S.N., Hook, M.A., and Miranda, R.C. (2006). Instrumental learning within the spinal cord: underlying mechanisms and implications for recovery after injury. Behav. Cogn. Neurosci. Rev. 5, 191-239.

10. Harkema, S., Gerasimenko, Y., Hodes, J., Burdick, J., Angeli, C., Chen, Y., Ferreira, C., Willhite, A., Rejc, E., Grossman, R.G., and Edgerton, V.R. (2011). Effect of epidural stimulation of the lumbosacral spinal cord on voluntary movement, standing, and assisted stepping after motor complete paraplegia: a case study. Lancet 377 , 1938-1947.

11. Ferguson, A.R., Stück, E.D., and Nielson, J.L. (2011). Syndromics: a bioinformatics approach for neurotrauma research. Transl. Stroke Res. 2, 438-454.

12. Awai, L., Bolliger, M., Ferguson, A.R., Courtine, G., and Curt, A. (2016). Influence of spinal cord integrity on gait control in human spinal cord injury. Neurorehabil. Neural Repair 30, 562-572.

13. Nielson, J.L., Paquette, J., Liu, A.W., Guandique, C.F., Tovar, C.A., Inoue, T., Irvine, K.-A., Gensel, J.C., Kloke, J., Petrossian, T.C., Lum, P.Y., Carlsson, G.E., Manley, G.T., Young, W., Beattie, M.S., Bresnahan, J.C., and Ferguson, A.R. (2015). Topological data analysis for discovery in preclinical spinal cord injury and traumatic brain injury. Nat. Commun. 6, 8581.

14. Dietz, V. (2012). Neuronal plasticity after a human spinal cord injury: positive and negative effects. Exp. Neurol. 235, 110-115.

15. Cha, J., Heng, C., Reinkensmeyer, D.J., Roy, R.R., Edgerton, V.R., and De Leon, R.D. (2007). Locomotor ability in spinal rats is dependent on the amount of activity imposed on the hindlimbs during treadmill training. J. Neurotrauma 24, 1000-1012.

16. Brown, T.G. (1911). The Intrinsic factors in the act of progression in the mammal. Proc. Royal Soc. B: Biol. Sci. 84, 308-319.

17. Brown, T.G. (1914). On the nature of the fundamental activity of the nervous centres; together with an analysis of the conditioning of rhythmic activity in progression, and a theory of the evolution of function in the nervous system. J. Physiol. (Lond.) 48, 18-46.

18. Sherrington, C.S. (1913). Further observations on the production of reflex stepping by combination of reflex excitation with reflex inhibition. J. Physiol. (Lond.) 47, 196-214.

19. Wilson, D.M., and Wyman, R.J. (1965). Motor output patterns during random and rhythmic stimulation of locust thoracic ganglia. Biophys. J. 5, 121-143.

20. Miller, S., and Scott, P.D. (1977). The spinal locomotor generator. Exp. Brain Res. 30, 387-403.

21. Stuart, D.G., and Hultborn, H. (2008). Thomas Graham Brown (1882-1965), Anders Lundberg (1920-), and the neural control of stepping. Brain Res. Rev. 59, 74-95.

22. Guertin, P.A. (2014). Preclinical evidence supporting the clinical development of central pattern generator-modulating therapies for chronic spinal cord-injured patients. Front. Hum. Neurosci. 8, 272.

23. Barbeau, H., and Rossignol, S. (1987). Recovery of locomotion after chronic spinalization in the adult cat. Brain Res. 412, 84-95.

24. Pearson, K.G., and Rossignol, S. (1991). Fictive motor patterns in chronic spinal cats. J. Neurophysiol. 66, 1874-1887.

25. Rossignol, S., Dubuc, R., and Gossard, J.-P. (2006). Dynamic sensorimotor interactions in locomotion. Physiol. Rev. 86, 89-154.

26. Frigon, A. (2012). Central pattern generators of the mammalian spinal cord. Neuroscientist 18, 56-69.

27. Grillner, S., and Zangger, P. (1979). On the central generation of locomotion in the low spinal cat. Exp. Brain Res. 34, 241-261.
28. Forssberg, H., Grillner, S., and Halbertsma, J. (1980). The locomotion of the low spinal cat. I. Coordination within a hindlimb. Acta Physiol. Scand. 108, 269-281.

29. Roy, R.R., Harkema, S.J., and Edgerton, V.R. (2012). Basic concepts of activity-based interventions for improved recovery of motor function after spinal cord injury. Arch. Phys Med. Rehabil. 93, 14871497.

30. Harkema, S.J., Hurley, S.L., Patel, U.K., Requejo, P.S., Dobkin, B.H., and Edgerton, V.R. (1997). Human lumbosacral spinal cord interprets loading during stepping. J. Neurophysiol. 77, 797-811.

31. Kojima, N., Nakazawa, K., and Yano, H. (1999). Effects of limb loading on the lower-limb electromyographic activity during orthotic locomotion in a paraplegic patient. Neurosci. Lett. 274, 211-213.

32. Ferris, D.P., Gordon, K.E., Beres-Jones, J.A., and Harkema, S.J. (2004). Muscle activation during unilateral stepping occurs in the nonstepping limb of humans with clinically complete spinal cord injury. Spinal Cord 42, 14-23.

33. García-Alías, G., Barkhuysen, S., Buckle, M., and Fawcett, J.W. (2009). Chondroitinase ABC treatment opens a window of opportunity for task-specific rehabilitation. Nat. Neurosci. 12, 1145-1151.

34. De Leon, R.D., Hodgson, J.A., Roy, R.R., and Edgerton, V.R. (1998). Full weight-bearing hindlimb standing following stand training in the adult spinal cat. J. Neurophysiol. 80, 83-91.

35. Bigbee, A.J., Crown, E.D., Ferguson, A.R., Roy, R.R., Tillakaratne, N.J.K., Grau, J.W., and Edgerton, V.R. (2007). Two chronic motor training paradigms differentially influence acute instrumental learning in spinally transected rats. Behav. Brain Res. 180, 95-101.

36. Forssberg, H., Grillner, S., and Rossignol, S. (1975). Phase dependent reflex reversal during walking in chronic spinal cats. Brain Res. $85,103-107$.

37. Forssberg, H. (1979). Stumbling corrective reaction: a phasedependent compensatory reaction during locomotion. J. Neurophysiol. 42, 936-953.

38. Bouyer, L.J.G., and Rossignol, S. (2003). Contribution of cutaneous inputs from the hindpaw to the control of locomotion. I. Intact cats. J. Neurophysiol. 90, 3625-3639.

39. Timoszyk, W.K., Nessler, J.A., Acosta, C., Roy, R.R., Edgerton, V.R., Reinkensmeyer, D.J., and de Leon, R. (2005). Hindlimb loading determines stepping quantity and quality following spinal cord transection. Brain Res. 1050, 180-189.

40. Shah, P.K., García-Alías, G., Choe, J., Gad, P., Gerasimenko, Y., Tillakaratne, N., Zhong, H., Roy, R.R., and Edgerton, V.R. (2013). Use of quadrupedal step training to re-engage spinal interneuronal networks and improve locomotor function after spinal cord injury. Brain 136, 3362-3377.

41. Juvin, L., Le Gal, J.-P., Simmers, J., and Morin, D. (2012). Cervicolumbar coordination in mammalian quadrupedal locomotion: role of spinal thoracic circuitry and limb sensory inputs. J. Neurosci. 32, 953-965.

42. Ward, P.J., Herrity, A.N., Harkema, S.J., and Hubscher, C.H. (2016), Training-induced functional gains following SCI. Neural Plast. 2016, 4307694.

43. Dominici, N., Keller, U., Vallery, H., Friedli, L., van den Brand, R., Starkey, M.L., Musienko, P., Riener, R., and Courtine, G. (2012). Versatile robotic interface to evaluate, enable and train locomotion and balance after neuromotor disorders. Nat. Med. 18, 1142-1147.

44. Hamlin, M., Traughber, T., Reinkensmeyer, D.J., and De Leon, R.D. (2015). A novel device for studying weight supported, quadrupedal overground locomotion in spinal cord injured rats. J. Neurosci. Methods 246, 134-141.

45. Dietz, V., Colombo, G., and Jensen, L. (1994). Locomotor activity in spinal man. Lancet 344, 1260-1263.

46. Duysens, J. (2002). Human gait as a step in evolution. Brain 125, 2589-2590.

47. Colombo, G., Joerg, M., Schreier, R., and Dietz, V. (2000). Treadmill training of paraplegic patients using a robotic orthosis. J. Rehabil. Res. Dev. 37, 693-700.

48. Wirz, M., Zemon, D.H., Rupp, R., Scheel, A., Colombo, G., Dietz, V., and Hornby, T.G. (2005). Effectiveness of automated locomotor training in patients with chronic incomplete spinal cord injury: a multicenter trial. Arch. Phys. Med. Rehabil 86, 672-680.

49. Field-Fote, E.C., Lindley, S.D., and Sherman, A.L. (2005). Locomotor training approaches for individuals with spinal cord injury: a preliminary report of walking-related outcomes. J. Neurol. Phys. Ther. 29, 127-137. 
50. Dietz, V. (2016). Clinical aspects for the application of robotics in locomotor neurorehabilitation, in: Neurorehabilitation Technology. D.J. Reinkensmeyer, and V. Dietz (eds). Springer International Publishing, pps. 209-222.

51. Hornby, T.G., Campbell, D.D., Kahn, J.H., Demott, T., Moore, J.L., and Roth, H.R. (2008). Enhanced gait-related improvements after therapist- versus robotic-assisted locomotor training in subjects with chronic stroke: a randomized controlled study. Stroke 39, 17861792.

52. Field-Fote, E.C., and Roach, K.E. (2011). Influence of a locomotor training approach on walking speed and distance in people with chronic spinal cord injury: a randomized clinical trial. Phys. Ther. 91, 48-60.

53. Kozlowski, A.J., Bryce, T.N., and Dijkers, M.P. (2015). Time and effort required by persons with spinal cord injury to learn to use a powered exoskeleton for assisted walking. Top. Spinal Cord Inj. Rehabil. 21, 110-121.

54. Jankowska, E., Jukes, M.G., Lund, S., and Lundberg, A. (1967). The effect of DOPA on the spinal cord. 6. Half-centre organization of interneurones transmitting effects from the flexor reflex afferents. Acta Physiol. Scand. 70, 389-402.

55. Chau, C., Barbeau, H., and Rossignol, S. (1998). Effects of intrathecal alpha1- and alpha2-noradrenergic agonists and norepinephrine on locomotion in chronic spinal cats. J. Neurophysiol. 79, 29412963.

56. Antri, M., Mouffle, C., Orsal, D., and Barthe, J.-Y. (2003). 5-HT1A receptors are involved in short- and long-term processes responsible for 5-HT-induced locomotor function recovery in chronic spinal rat. Eur. J. Neurosci. 18, 1963-1972.

57. Fong, A.J., Cai, L.L., Otoshi, C.K., Reinkensmeyer, D.J., Burdick, J.W., Roy, R.R., and Edgerton, V.R. (2005). Spinal cord-transected mice learn to step in response to quipazine treatment and robotic training. J. Neurosci. 25, 11738-11747.

58. Barbeau, H., and Rossignol, S. (1991). Initiation and modulation of the locomotor pattern in the adult chronic spinal cat by noradrenergic, serotonergic and dopaminergic drugs. Brain Res. 546, 250-260.

59. Courtine, G., Gerasimenko, Y., van den Brand, R., Yew, A., Musienko, P., Zhong, H., Song, B., Ao, Y., Ichiyama, R.M., Lavrov, I., Roy, R.R., Sofroniew, M.V., and Edgerton, V.R. (2009). Transformation of nonfunctional spinal circuits into functional states after the loss of brain input. Nat. Neurosci. 12, 1333-1342.

60. Buehner, J.J., Forrest, G.F., Schmidt-Read, M., White, S., Tansey, K., and Basso, D.M. (2012). Relationship between ASIA examination and functional outcomes in the NeuroRecovery Network Locomotor Training Program. Arch. Phys. Med. Rehabil. 93, 1530 1540 .

61. Hansen, C.N., Faw, T.D., White, S., Buford, J.A., Grau, J.W., and Basso, D.M. (2016). Sparing of descending axons rescues interneuron plasticity in the lumbar cord to allow adaptive learning after thoracic spinal cord injury. Front. Neural Circuits 10, 11.

62. Rank, M.M., Flynn, J.R., Galea, M.P., Callister, R., and Callister, R.J. (2015). Electrophysiological characterization of spontaneous recovery in deep dorsal horn interneurons after incomplete spinal cord injury. Exp. Neurol. 271, 468-478.

63. Mills, C.D., Grady, J.J., and Hulsebosch, C.E. (2001). Changes in exploratory behavior as a measure of chronic central pain following spinal cord injury. J. Neurotrauma 18, 1091-1105.

64. Finnerup, N.B., Johannesen, I.L., Fuglsang-Frederiksen, A., Bach, F.W., and Jensen, T.S. (2003). Sensory function in spinal cord injury patients with and without central pain. Brain 126, 57-70.

65. Gómez-Pinilla, F., Ying, Z., Roy, R.R., Molteni, R., and Edgerton, V.R. (2002). Voluntary exercise induces a BDNF-mediated mechanism that promotes neuroplasticity. J. Neurophysiol. 88, 2187-2195.

66. Hutchinson, K.J., Gómez-Pinilla, F., Crowe, M.J., Ying, Z., and Basso, D.M. (2004). Three exercise paradigms differentially improve sensory recovery after spinal cord contusion in rats. Brain 127, 14031414.

67. Brown, A.K., Woller, S.A., Moreno, G., Grau, J.W., and Hook, M.A. (2011). Exercise therapy and recovery after SCI: evidence that shows early intervention improves recovery of function. Spinal Cord 49, 623-628.

68. Hansen, C.N., Fisher, L.C., Deibert, R.J., Jakeman, L.B., Zhang, H., Noble-Haeusslein, L., White, S., and Basso, D.M. (2013). Elevated MMP-9 in the lumbar cord early after thoracic spinal cord injury impedes motor relearning in mice. J. Neurosci. 33, 13101-13111.
69. Detloff, M.R., Smith, E.J., Quiros Molina, D., Ganzer, P.D., and Houlé, J.D. (2014). Acute exercise prevents the development of neuropathic pain and the sprouting of non-peptidergic (GDNF- and arteminresponsive) c-fibers after spinal cord injury. Exp. Neurol. 255, 38-48.

70. Detloff, M.R., Quiros Molina, D., Javia, A.S., Daggubati, L., Nehlsen, A.D., Naqvi, A., Ninan, V., Vannix, K.N., McMullen, M.-K., Amin, S., Ganzer, P.D., and Houlé, J.D. (2015). Delayed exercise is ineffective at reversing aberrant nociceptive afferent plasticity or neuropathic pain after spinal cord injury in rats. Neurorehabil. Neural Repair 30, 685-700.

71. Nees, T.A., Tappe-Theodor, A., Sliwinski, C., Motsch, M., Rupp, R., Kuner, R., Weidner, N., and Blesch, A. (2016). Early-onset treadmill training reduces mechanical allodynia and modulates calcitonin gene-related peptide fiber density in lamina III/IV in a mouse model of spinal cord contusion injury. Pain 157, 687-697.

72. Battistuzzo, C.R., Callister, R.J., Callister, R., and Galea, M.P. (2012). A systematic review of exercise training to promote locomotor recovery in animal models of spinal cord injury. J. Neurotrauma 29, 1600-1613.

73. Leasure, J.L., and Schallert, T. (2004). Consequences of forced disuse of the impaired forelimb after unilateral cortical injury. Behav. Brain Res. 150, 83-91.

74. Hansen, C.N., Fisher, L.C., Deibert, R.J., Jakeman, L.B., Zhang, H., Noble-Haeusslein, L., White, S., and Basso, D.M. (2013). Elevated MMP-9 in the lumbar cord early after thoracic spinal cord injury impedes motor relearning in mice. J. Neurosci. 33, 13101-13111.

75. Griesbach, G.S., Gómez-Pinilla, F., and Hovda, D.A. (2007). Time window for voluntary exercise-induced increases in hippocampal neuroplasticity molecules after traumatic brain injury is severity dependent. J. Neurotrauma 24, 1161-1171.

76. Hutchinson, K.J., Linderman, J.K., and Basso, D.M. (2001). Skeletal muscle adaptations following spinal cord contusion injury in rat and the relationship to locomotor function: a time course study. J. Neurotrauma 18, 1075-1089.

77. Liu, M., Bose, P., Walter, G.A., Thompson, F.J., and Vandenborne, K. (2008). A longitudinal study of skeletal muscle following spinal cord injury and locomotor training. Spinal Cord 46, 488-493.

78. Ohnishi, Y.-I., Iwatsuki, K., Shinzawa, K., Nakai, Y., Ishihara, M., and Yoshimine, T. (2012). Disuse muscle atrophy exacerbates motor neuronal degeneration caudal to the site of spinal cord injury. Neuroreport 23, 157-161.

79. Ye, F., Baligand, C., Keener, J.E., Vohra, R., Lim, W., Ruhella, A., Bose, P., Daniels, M., Walter, G.A., Thompson, F., and Vandenborne, K. (2013). Hindlimb muscle morphology and function in a new atrophy model combining spinal cord injury and cast immobilization. J. Neurotrauma 30, 227-235.

80. Yarrow, J.F., Ye, F., Balaez, A., and Mantione, J.M. (2014). Bone loss in a new rodent model combining spinal cord injury and cast immobilization. J. Musculoskelet. Neuronal Interact. 14, 255-266.

81. Dudley-Javoroski, S., and Shields, R.K. (2008). Muscle and bone plasticity after spinal cord injury: review of adaptations to disuse and to electrical muscle stimulation. J. Rehabil. Res. Dev. 45, 283-296.

82. Grau, J.W., Washburn, S.N., Hook, M.A., Ferguson, A.R., Crown, E.D., Garcia, G., Bolding, K.A., and Miranda, R.C. (2004). Uncontrollable stimulation undermines recovery after spinal cord injury. J. Neurotrauma 21, 1795-1817

83. Ferguson, A.R., Huie, J.R., Crown, E.D., and Grau, J.W. (2012). Central nociceptive sensitization vs. spinal cord training: opposing forms of plasticity that dictate function after complete spinal cord injury. Front. Physiol. 3, 396.

84. Caudle, K.L., Brown, E.H., Shum-Siu, A., Burke, D.A., Magnuson, T.S.G., Voor, M.J., and Magnuson, D.S.K. (2011). Hindlimb immobilization in a wheelchair alters functional recovery following contusive spinal cord injury in the adult rat. Neurorehabil. Neural Repair 25, 729-739.

85. Morey-Holton, E.R., and Globus, R.K. (2002). Hindlimb unloading rodent model: technical aspects. J. Appl. Physiol. 92, 1367-1377.

86. Globus, R.K., and Morey-Holton, E. (2016). Hindlimb unloading: rodent analog for microgravity. J. Appl. Physiol. (1985) 120, 11961206.

87. Edgerton, V.R., Wolf, S.L., Levendowski, D.J., and Roy, R.R. (1996). Theoretical basis for patterning EMG amplitudes to assess muscle dysfunction. Med. Sci. Sports Exerc. 28, 744-751.

88. De-Doncker, L., Kasri, M., Picquet, F., and Falempin, M. (2005). Physiologically adaptive changes of the L5 afferent neurogram and 
of the rat soleus EMG activity during 14 days of hindlimb unloading and recovery. J. Exp. Biol. 208, 4585-4592.

89. De-Doncker, L., Kasri, M., and Falempin, M. (2006). Soleus motoneuron excitability after rat hindlimb unloading using histology and a new electrophysiological approach to record a neurographic analogue of the H-reflex. Exp. Neurol. 201, 368-374.

90. Takeoka, A., Vollenweider, I., Courtine, G., and Arber, S. (2014). Muscle spindle feedback directs locomotor recovery and circuit reorganization after spinal cord injury. Cell 159, 1626-1639.

91. Anderson, J., Almeida-Silveira, M.I., and Pérot, C. (1999). Reflex and muscular adaptations in rat soleus muscle after hindlimb suspension. J. Exp. Biol. 202, 2701-2707.

92. De-Doncker, L., Kasri, M., and Falempin, M. (2006). Soleus motoneuron excitability after rat hindlimb unloading using histology and a new electrophysiological approach to record a neurographic analogue of the H-reflex. Exp. Neurol. 201, 368-374.

93. Misiaszek, J.E. (2003). The H-reflex as a tool in neurophysiology: its limitations and uses in understanding nervous system function. Muscle Nerve 28, 144-160.

94. Canu, M.H., and Falempin, M. (1998). Effect of hindlimb unloading on interlimb coordination during treadmill locomotion in the rat. Eur. J. Appl. Physiol. Occup. Physiol. 78, 509-515.

95. Ruegg, D.G., Kakebeeke, T.H., Gabriel, J.-P., and Bennefeld, M. (2003). Conduction velocity of nerve and muscle fiber action potentials after a space mission or a bed rest. Clin. Neurophysiol. 114, 86-93.

96. Clark, B.C., Manini, T.M., Bolanowski, S.J., and Ploutz-Snyder, L.L. (2006). Adaptations in human neuromuscular function following prolonged unweighting: II. Neurological properties and motor imagery efficacy. J. Appl. Physiol. 101, 264-272.

97. Morioka, Tazoe, Ma, Guandique, VanCitters, Huie, Bresnahan, Beattie, Tanaka, Ferguson, \& Ogata (2015). Early hindlimb unloading produces maladaptive plasticity that limits functional recovery after spinal cord injury (SCI). Journal of Neurotrauma. June 2014, 31(12): A-1-A-126. doi:10.1089/neu.2014.9935.abstracts.

98. Saboe, L.A., Reid, D.C., Davis, L.A., Warren, S.A., and Grace, M.G. (1991). Spine trauma and associated injuries. J. Trauma 31, 43-48.

99. Wang, C.M., Chen, Y., DeVivo, M.J., and Huang, C.T. (2001). Epidemiology of extraspinal fractures associated with acute spinal cord injury. Spinal Cord 39, 589-594.

100. Sekhon, L.H., and Fehlings, M.G. (2001). Epidemiology, demographics, and pathophysiology of acute spinal cord injury. Spine 26, S2-S12.

101. Hasler, R.M., Exadaktylos, A.K., Bouamra, O., Benneker, L.M., Clancy, M., Sieber, R., Zimmermann, H., and Lecky, F. (2011). Epidemiology and predictors of spinal injury in adult major trauma patients: European cohort study. Eur. Spine J. 20, 2174-2180.

102. Sandkuhler, J., and Liu, X. (1998). Induction of long-term potentiation at spinal synapses by noxious stimulation or nerve injury. Eur. J. Neurosci. 10, 2476-2480.

103. Ikeda, H. (2006). Synaptic amplifier of inflammatory pain in the spinal dorsal horn. Science 312, 1659-1662.

104. Tan, A.M., and Waxman, S.G. (2012). Spinal cord injury, dendritic spine remodeling, and spinal memory mechanisms. Exp. Neurol. $235,142-151$.

105. Ji, R.-R., Kohno, T., Moore, K.A., and Woolf, C.J. (2003). Central sensitization and LTP: do pain and memory share similar mechanisms? Trends Neurosc. 26, 696-705.

106. Sandkuhler, J. (2009). Models and mechanisms of hyperalgesia and allodynia. Physiol. Rev. 89, 707-758.

107. Ruscheweyh, R., Wilder-Smith, O., Drdla, R., Liu, X.-G., and Sandkühler, J. (2011). Long-term potentiation in spinal nociceptive pathways as a novel target for pain therapy. Mol. Pain 7, 20.

108. Drdla-Schutting, R., Benrath, J., Wunderbaldinger, G., and Sandkühler, J. (2012). Erasure of a spinal memory trace of pain by a brief, high-dose opioid administration. Science 335, 235-238.

109. Grau, J.W., Barstow, D.G., and Joynes, R.L. (1998). Instrumental learning within the spinal cord: I. Behavioral properties. Behav. Neurosci. 112, 1366-1386.

110. Garraway, S.M., Turtle, J.D., Huie, J.R., Lee, K.H., Hook, M.A., Woller, S.A., and Grau, J.W. (2011). Intermittent noxious stimulation following spinal cord contusion injury impairs locomotor recovery and reduces spinal brain-derived neurotrophic factortropomyosin-receptor kinase signaling in adult rats. Neuroscience 199, 86-102.
111. Huie, J.R., Garraway, S.M., Baumbauer, K.M., Hoy, K.C., Beas, B.S., Montgomery, K.S., Bizon, J.L., and Grau, J.W. (2012). Brainderived neurotrophic factor promotes adaptive plasticity within the spinal cord and mediates the beneficial effects of controllable stimulation. Neuroscience 200, 74-90.

112. Grau, J.W., Huie, J.R., Garraway, S.M., Hook, M.A., Crown, E.D., Baumbauer, K.M., Lee, K.H., Hoy, K.C., and Ferguson, A.R. (2012) Impact of behavioral control on the processing of nociceptive stimulation. Front. Physiol. 3, 262.

113. Garraway, S.M., Woller, S.A., Huie, J.R., Hartman, J.J., Hook, M.A., Miranda, R.C., Huang, Y.-J., Ferguson, A.R., and Grau, J.W. (2014). Peripheral noxious stimulation reduces withdrawal threshold to mechanical stimuli after spinal cord injury: role of tumor necrosis factor alpha and apoptosis. Pain 155, 2344-2359.

114. Bouffard, J., Bouyer, L.J., Roy, J.-S., and Mercier, C. (2014), Tonic pain experienced during locomotor training impairs retention despite normal performance during acquisition. J. Neurosci. 34, 9190-9195.

115. Ferguson, A.R., Crown, E.D., and Grau, J.W. (2006). Nociceptive plasticity inhibits adaptive learning in the spinal cord. Neuroscience $141,421-431$.

116. Crown, E.D., Ferguson, A.R., Joynes, R.L., and Grau, J.W. (2002). Instrumental learning within the spinal cord. II. Evidence for central mediation. Physiol. Behav. 77, 259-267.

117. Baumbauer, K.M., Hoy, K.C., Huie, J.R., Hughes, A.J., Woller, S.A., Puga, D.A., Setlow, B., and Grau, J.W. (2008). Timing in the absence of supraspinal input I: variable, but not fixed, spaced stimulation of the sciatic nerve undermines spinally-mediated instrumental learning. Neuroscience 155, 1030-1047.

118. Huie, J.R., Stück, E.D., Lee, K.H., Irvine, K.-A., Beattie, M.S., Bresnahan, J.C., Grau, J.W., and Ferguson, A.R. (2015). AMPA receptor phosphorylation and synaptic colocalization on motor neurons drive maladaptive plasticity below complete spinal cord injury. eNeuro 2, pii:ENEURO.0091-15.2015.

119. Hartmann, B., Ahmadi, S., Heppenstall, P.A., Lewin, G.R., Schott, C., Borchardt, T., Seeburg, P.H., Zeilhofer, H.U., Sprengel, R., and Kuner, R. (2004). The AMPA receptor subunits GluR-A and GluR-B reciprocally modulate spinal synaptic plasticity and inflammatory pain. Neuron 44, 637-650.

120. Galan, A., Laird, J.M.A., and Cervero, F. (2004). In vivo recruitment by painful stimuli of AMPA receptor subunits to the plasma membrane of spinal cord neurons. Pain 112, 315-323.

121. Ferguson, A.R., Christensen, R.N., Gensel, J.C., Miller, B.A., Sun, F., Beattie, E.C., Bresnahan, J.C., and Beattie, M.S. (2008). Cell death after spinal cord injury is exacerbated by rapid TNF alpha-induced trafficking of GluR2-lacking AMPARs to the plasma membrane. J. Neurosci. 28, 11391-11400.

122. Van Damme, P., Callewaert, G., Eggermont, J., Robberecht, W., and Van Den Bosch, L. (2003). Chloride influx aggravates Ca2+-dependent AMPA receptor-mediated motoneuron death. J. Neurosci. 23, 49424950.

123. Liu, Y., Wang, L., Long, Z.-Y., Wu, Y.-M., Wan, Q., Jiang, J.-X., and Wang, Z.-G. (2013). Inhibiting PTEN protects hippocampal neurons against stretch injury by decreasing membrane translocation of AMPA receptor GluR2 subunit. PLoS One 8, e65431.

124. Abraham, W.C., and Bear, M.F. (1996). Metaplasticity: the plasticity of synaptic plasticity. Trends Neurosci. 19, 126-130.

125. Ireland, D.R., and Abraham, W.C. (2002). Group I mGluRs increase excitability of hippocampal CA1 pyramidal neurons by a PLCindependent mechanism. J. Neurophysiol. 88, 107-116.

126. Oh, M.C., Derkach, V.A., Guire, E.S., and Soderling, T.R. (2006). Extrasynaptic membrane trafficking regulated by GluR1 serine 845 phosphorylation primes AMPA receptors for long-term potentiation. J. Biol. Chem. 281, 752-758.

127. Sajikumar, S., and Korte, M. (2011). Metaplasticity governs compartmentalization of synaptic tagging and capture through brainderived neurotrophic factor (BDNF) and protein kinase Mzeta (PKMzeta). Proc. Natl. Acad. Sci. U. S. A. 108, 2551-2556.

128. Parker, D., and Grillner, S. (1999). Activity-dependent metaplasticity of inhibitory and excitatory synaptic transmission in the lamprey spinal cord locomotor network. J. Neurosci. 19, 1647-1656.

129. Bevan, S., and Parker, D. (2004). Metaplastic facilitation and ultrastructural changes in synaptic properties are associated with longterm modulation of the lamprey locomotor network. J. Neurosci. 24, 9458-9468. 
130. Lee, J.K., Emch, G.S., Johnson, C.S., and Wrathall, J.R. (2005). Effect of spinal cord injury severity on alterations of the H-reflex. Exp. Neurol. 196, 430-440.

131. Ferguson, A.R., Bolding, K.A., Huie, J.R., Hook, M.A., Santillano, D.R., Miranda, R.C., and Grau, J.W. (2008). Group I metabotropic glutamate receptors control metaplasticity of spinal cord learning through a protein kinase C-dependent mechanism. J. Neurosci. 28 11939-11949.

132. Huie, J.R., Baumbauer, K.M., Lee, K.H., Bresnahan, J.C., Beattie, M.S., Ferguson, A.R., and Grau, J.W. (2012). Glial tumor necrosis factor alpha $(\mathrm{TNF} \alpha)$ generates metaplastic inhibition of spinal learning. PLoS One 7, e39751.

133. Sorkin, L.S., Yaksh, T.L., and Doom, C.M. (1999). Mechanical allodynia in rats is blocked by a $\mathrm{Ca} 2+$ permeable AMPA receptor antagonist. Neuroreport 10, 3523-3526.

134. Sorkin, L.S., Yaksh, T.L., and Doom, C.M. (2001). Pain models display differential sensitivity to Ca2+-permeable non-NMDA glutamate receptor antagonists. Anesthesiology 95, 965-973.

135. Walters, M.R., Kaste, M., Lees, K.R., Diener, H.C., Hommel, M., De Keyser, J., Steiner, H., and Versavel, M. (2005). The AMPA antagonist ZK 200775 in patients with acute ischaemic stroke: a double-blind, multicentre, placebo-controlled safety and tolerability study. Cerebrovasc. Dis. 20, 304-309.

136. Leonoudakis, D., Zhao, P., and Beattie, E.C. (2008). Rapid tumor necrosis factor alpha-induced exocytosis of glutamate receptor 2lacking AMPA receptors to extrasynaptic plasma membrane potentiates excitotoxicity. J. Neurosci. 28, 2119-2130.

137. Spaethling, J.M., Klein, D.M., Singh, P., and Meaney, D.F. (2008). Calcium-permeable AMPA receptors appear in cortical neurons after traumatic mechanical injury and contribute to neuronal fate. $\mathrm{J}$. Neurotrauma 25, 1207-1216.

138. Yin, H.Z., Hsu, C.-I., Yu, S., Rao, S.D., Sorkin, L.S., and Weiss, J.H. (2012). TNF- $\alpha$ triggers rapid membrane insertion of $\mathrm{Ca}(2+)$ permeable AMPA receptors into adult motor neurons and enhances their susceptibility to slow excitotoxic injury. Exp. Neurol. 238, 93-102.

139. Dias, R.B., Rombo, D.M., Ribeiro, J.A., and Sebastião, A.M. (2013) Ischemia-induced synaptic plasticity drives sustained expression of calcium-permeable AMPA receptors in the hippocampus. Neuropharmacology 65, 114-122.

140. Cabañero, D., Baker, A., Zhou, S., Hargett, G.L., Irie, T., Xia, Y. Beaudry, H., Gendron, L., Melyan, Z., Carlton, S.M., and Morón, J.A. (2013). Pain after discontinuation of morphine treatment is associated with synaptic increase of GluA4-containing AMPAR in the dorsal horn of the spinal cord. Neuropsychopharmacology 38, 14721484.

141. Stellwagen, D., and Malenka, R.C. (2006). Synaptic scaling mediated by glial TNF-alpha. Nature 440, 1054-1059.

Address correspondence to:

Adam R. Ferguson, PhD

Brain and Spinal Injury Center

University of California, San Francisco

1001 Potrero Avenue, Building 1, Room 101

San Francisco, CA 94110

E-mail: Adam.ferguson@ucsf.edu 